\title{
Safety and efficacy of panitumumab therapy after metastatic colorectal cancer progression with cetuximab: Experience at a single Japanese institution
}

\author{
HIROMICHI SONODA, EIJI MEKATA, TOMOHARU SHIMIZU, \\ YOSHIHIRO ENDO and TOHRU TANI \\ Department of Surgery, Shiga University of Medical Science, Shiga 520-2192, Japan
}

Received October 28, 2012; Accepted January 28, 2013

DOI: $10.3892 / \mathrm{ol} .2013 .1171$

\begin{abstract}
Panitumumab (Pmab) is generally considered to be ineffective after the failure of cetuximab ( $\mathrm{Cmab})$ therapy in metastatic colorectal cancer (mCRC) patients. However, a few studies have demonstrated that Pmab is an effective treatment for disease progression following Cmab-based regimens in the USA. In the present study, we evaluated the safety and efficacy of Pmab therapy following the failure of Cmab therapy in Japanese patients with mCRC. We performed a retrospective review of the treatment of 16 mCRC patients who tolerated Pmab with clinical benefits after the failure of Cmab therapy between August 2010 and September 2011 at Shiga University of Medical Science. Fourteen of the 16 patients were administered standard Pmab monotherapy $(6 \mathrm{mg} / \mathrm{kg})$ intravenously every 2 weeks and the remaining two patients received Pmab with mFOLFOX6 intravenously every 2 weeks. All patients received Pmab chemotherapy until the occurrence of disease progression. Partial radiographic responses (PR) were observed in 2 of the 16 patients and stable disease (SD) was observed in 5 patients. Nine patients had evidence of progressive disease (PD). According to the KRAS status, 7 of the $13(53.8 \%)$ patients who had wild-type $K R A S$ achieved a high disease control rate $(\mathrm{PR}+\mathrm{SD})$. The median progression-free survival (PFS) and overall survival (OS) in the wild-type $K R A S$ patients was 96 and 245 days, respectively. Pmab may be an alternative treatment strategy for Japanese patients with mCRC who have experienced failure with standard Cmabbased therapeutic regimens.
\end{abstract}

Correspondence to: Dr Hiromichi Sonoda, Department of Surgery, Shiga University of Medical Science, Seta Tsukinowa-cho, Otsu, Shiga 520-2192, Japan

E-mail: hirosono@belle.shiga-med.ac.jp

Key words: anti-epidermal growth factor receptor therapy, panitumumab, monoclonal antibodies, colorectal cancer, chemotherapy

\section{Introduction}

Cetuximab (Cmab) is an anti-epidermal growth factor receptor (EGFR) antibody that has been shown to effectively combine with cytotoxic chemotherapy as first-, second- or third-line treatment against wild-type KRAS colorectal cancer (1-3). However, it has occasionally been associated with the development of hypersensitivity reactions (HSRs). In patients with severe HSRs, further therapy with Cmab is not possible. Compared with Cmab, HSRs have rarely been observed with panitumumab (Pmab), a fully human IgG anti-EGFR antibody (4). Pmab is considered to be ineffective in patients who experience failure with Cmab therapy, due to evidence that the two antibodies target the same receptor. However, a few studies have indicated that Pmab is effective in patients with refractory metastatic colorectal cancer (mCRC) following the failure of standard therapy, including Cmab-based regimens, in the USA $(5,6)$. However, there are no studies on the efficacy of Pmab therapy after the failure of Cmab therapy in patients from Asian countries. In this study, we aimed to reveal the safety and efficacy of Pmab therapy following disease progression with Cmab therapy in Japanese mCRC patients.

\section{Patients and methods}

Patient information. We retrospectively reviewed $16 \mathrm{mCRC}$ patients who tolerated Pmab with clinical benefits after the failure of Cmab therapy between August 2010 and September 2011 at Shiga University of Medical Science. Patient medical records were reviewed for previous therapy, toxicity and response assessment. KRAS status was retrospectively assessed in patients with readily available tumor tissues. Chemotherapeutic response was assessed using the Response Evaluation Criteria in Solid Tumors (RECIST) (7). The incidence and severity of adverse events (AEs) were measured throughout the study and graded using the National Cancer Institute Common Toxicity Criteria version 4.0 (NCI-CTCAE v4.0).

This study protocol was in accordance with the ethical guidelines established by the Declaration of Helsinki. Written informed consent was obtained from all patients. 
Statistical analysis. Progression-free survival (PFS) and overall survival (OS) were calculated as the interval between the first day of Pmab treatment and the date of proven recurrence or death from any cause, respectively. PFS and OS were estimated using the Kaplan-Meier method.

\section{Results}

Patient characteristics. The baseline characteristics of the patient population are summarized in Table I. Thirteen patients were male and three were female. The median age was 65 years (range, 53-88 years). All patients had an ECOG (European Clinical Oncology Group) performance status between 0 and 2 . The site of the primary tumor was the colon in 5 of the 16 patients (31\%) and the rectum in 11 patients $(69 \%)$. Histologically, the primary tumor was a well-differentiated adenocarcinoma in 6 and a moderately differentiated adenocarcinoma in 10 patients. Ten of the 16 patients (63\%) had only one metastatic site (the lungs in 9 cases and liver in 1 case) and 6 of the 16 patients (38\%) had two or more metastatic sites, including the liver (5 patients), lungs (5 patients) and lymph nodes ( 2 patients). Thirteen of the 16 patients (81\%) had wild-type KRAS and three (19\%) had mutant-type $K R A S$. The median number of previous therapies was three (range, 2-6 therapies). Seven of the 16 patients (44\%) received four or more previous therapies. All patients received prior irinotecan therapy. Fifteen patients (94\%) received prior oxaliplatin and 14 patients $(88 \%)$ received prior bevacizumab therapy. All patients had been previously treated with Cmab and only two of the 16 patients (13\%) discontinued Cmab therapy due to the development of HSRs. Other reasons for stopping Cmab therapy included disease progression $(\mathrm{n}=13,81 \%)$ and the inconvenience of a bi-weekly schedule $(\mathrm{n}=1,6 \%)$. Fourteen of the 16 patients $(88 \%)$ received standard Pmab monotherapy $(6 \mathrm{mg} / \mathrm{kg})$ intravenously every 2 weeks, while the remaining two $(13 \%)$ received Pmab with mFOLFOX6 intravenously every 2 weeks. All patients in this study were administered standard medications (i.e., corticosteroids and antihistamines) prior to Pmab administration in order to prevent HSRs.

Therapeutic effect. All patients received Pmab chemotherapy until disease progression occurred. The median number of Pmab cycles administered was 7 (range, 3-15). Partial radiographic responses (PR) were noted in 2 of the 16 patients (12.5\%) and stable disease (SD) in 5 patients $(31.3 \%)$. Nine patients $(56.3 \%)$ had evidence of progressive disease (PD). In terms of KRAS status, all 3 patients with mutant-type $K R A S$ had evidence of PD and 7 of the 13 patients with wild-type KRAS (53.8\%) achieved a high disease control rate (PR $+\mathrm{SD})$. The median PFS and OS in patients with wild-type KRAS was 96 (Fig. 1) and 245 days (Fig. 2), respectively.

Carcinoembryonic antigen (CEA) levels. One patient did not exhibit a change in CEA level, regardless of tumor status. Four patients achieved a $>50 \%$ reduction ( 4934.5 to $793 \mathrm{U} / 1,266.4$ to $58.3 \mathrm{U} / 1,218.2$ to $55 \mathrm{U} / 1$ and 31.8 to $14.5 \mathrm{U} / \mathrm{l}), 2$ patients had a $25 \%$ reduction ( 459.5 to $296.5 \mathrm{U} / 1$ and 34.7 to $22.7 \mathrm{U} / \mathrm{l}$ ) and 1 patient had a minor reduction (442.8 to $380 \mathrm{U} / 1)$ in CEA levels and 8 patients had increased CEA levels. Therefore,
Table I. Baseline and clinical characteristics of the metastatic colorectal cancer patients.

\begin{tabular}{|c|c|}
\hline Characteristics & Value \\
\hline Total number (\%) & $16(100)$ \\
\hline Male/female (\%) & $13 / 3(81 / 19)$ \\
\hline \multicolumn{2}{|l|}{ Age (years) } \\
\hline Median & 65 \\
\hline Range & $53-88$ \\
\hline \multicolumn{2}{|l|}{ Performance status (\%) } \\
\hline 0 & $7(44)$ \\
\hline 1 & $5(31)$ \\
\hline 2 & $4(25)$ \\
\hline \multicolumn{2}{|l|}{ Primary tumor site $(\%)$} \\
\hline Colon & $5(31.25)$ \\
\hline Rectum & $11(68.75)$ \\
\hline \multicolumn{2}{|l|}{ Histology (\%) } \\
\hline Well-differentiated & $6(37.5)$ \\
\hline Moderately differentiated & $10(62.5)$ \\
\hline Poorly differentiated and others & $0(0)$ \\
\hline \multicolumn{2}{|l|}{ Number of metastatic sites (\%) } \\
\hline 1 & $10(62)$ \\
\hline 2 & $3(19)$ \\
\hline$\geq 3$ & $3(19)$ \\
\hline \multicolumn{2}{|l|}{ Sites of metastases (\%) } \\
\hline Liver & $6(38)$ \\
\hline Lungs & $14(88)$ \\
\hline Lymph nodes & $2(13)$ \\
\hline Other & $5(31)$ \\
\hline \multicolumn{2}{|l|}{$K R A S$ status (\%) } \\
\hline Wild-type & $13(81)$ \\
\hline Mutant & $3(19)$ \\
\hline \multicolumn{2}{|l|}{ Prior therapeutic regimens (\%) } \\
\hline 2 & $6(38)$ \\
\hline 3 & $3(19)$ \\
\hline$\geq 4$ & $7(44)$ \\
\hline Prior oxaliplatin therapy (\%) & $15(94)$ \\
\hline Prior irinotecan therapy (\%) & $16(100)$ \\
\hline Prior bevacizumab therapy (\%) & $14(88)$ \\
\hline
\end{tabular}

approximately half of the patients achieved a reduction in CEA levels.

Typical case. A 73-year-old male had been diagnosed with sigmoid colon cancer (T3N1M0 Stage IIIa) 7 years previously, for which he underwent a sigmoidectomy with regional lymph node excision. Two years after sigmoidectomy, the patient underwent partial hepatectomy for solitary liver metastasis. The patient was once again diagnosed with multiple liver metastases several months after the partial hepatectomy, for which hepatic arterial infusion chemotherapy (HAI) was administered. However, due to disease progression despite 


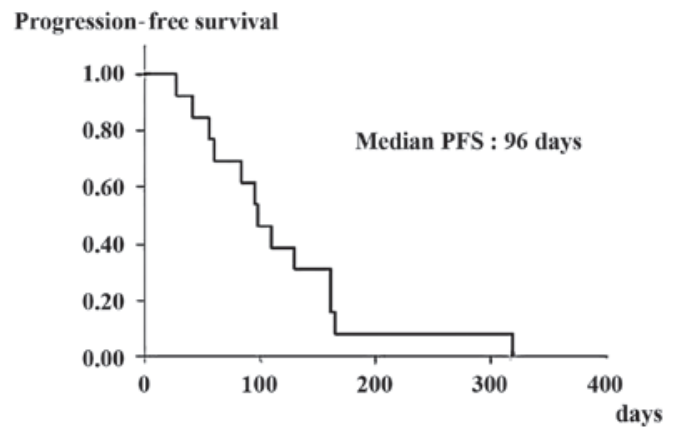

Figure 1. Kaplan-Meier curve demonstrating a median progression-free survival (PFS) of 96 days in patients with wild-type KRAS.



Figure 2. Kaplan-Meier curve demonstrating a median overall survival (OS) of 245 days in patients with wild-type $K R A S$.
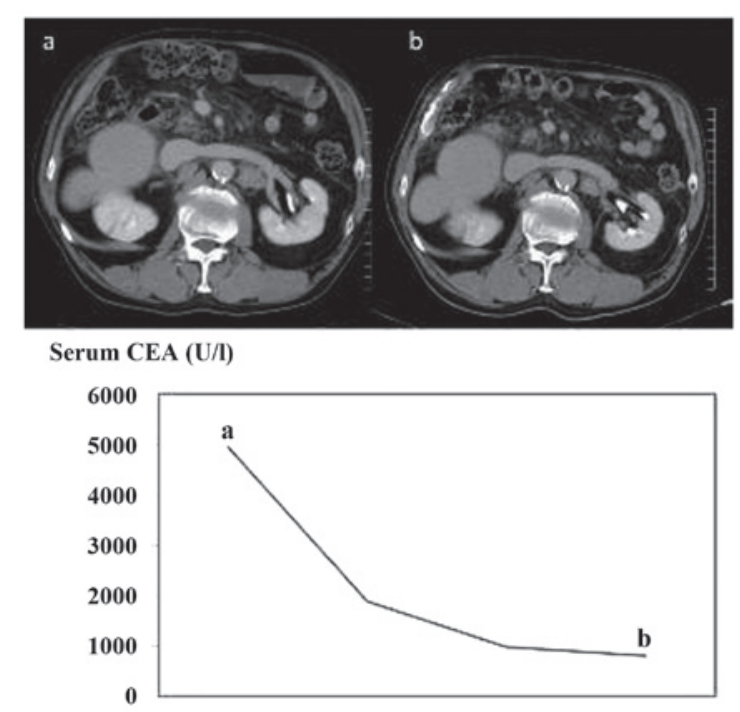

Figure 3. Patient with unique sensitivity to Pmab after various therapies, including cetuximab (Cmab). (a) Before Pmab treatment; (b) 10 months after Pmab treatment. CEA, carcinoembryonic antigen.

the HAI, mFOLFOX6, FOLFIRI and Cmab chemotherapy was administered. Thereafter, the patient was also diagnosed with lung and intra-abdominal lymph node metastases and received Pmab therapy, following which disease stability was achieved for $\sim 10$ months and CEA levels decreased from 4934 to $793 \mathrm{U} / 1$ (Fig. 3).
AEs. All patients tolerated Pmab well, with no cases of HSR. Grade 3/4 toxicities included hypomagnesemia in 1 patient and hypocalcemia in another. Other AEs observed included grade 1-2 skin rash in 3 patients, grade 2 hypomagnesemia in 2 patients, grade 1-2 fatigue in 3 patients and grade 1-2 appetite loss in 3 patients. However, none of the patients required discontinuation of Pmab therapy as a result of these AEs.

\section{Discussion}

For $\sim 40$ years, fluoropyrimidine 5-fluorouracil was the only drug available for the treatment of mCRC. However, over the past 10 years, the therapeutic armamentarium for $\mathrm{mCRC}$ has expanded significantly with the development and approval of three cytotoxic agents, irinotecan, oxaliplatin and the oral fluoropyrimidine capecitabine (8). During this time, three new biological agents were developed, including the anti-vascular endothelial growth factor (VEGF) antibody bevacizumab and anti-EGFR antibodies $\mathrm{Cmab}$ and Pmab. Incorporation of these biological agents into various cytotoxic chemotherapy regimens has transformed $\mathrm{mCRC}$ into a chronic illness, where the median OS is currently 24-28 months (9).

Cmab and Pmab bind with a high affinity to EGFRs and prevent the binding of natural growth factor ligands to these receptors (10). This inhibitory effect leads to the repression of subsequent downstream signaling pathways, which mediate cell growth and proliferation, survival mechanisms against chemotherapy and/or radiation therapy, invasion/metastasis and even angiogenesis. Cmab or Pmab therapy is not considered to be effective following disease progression with Pmab or Cmab therapy, respectively. However, Cmab is a chimeric antibody that is associated with the development of HSRs. The development of severe HSRs means premature termination of drug therapy is necessary $(11,12)$. Pmab is a fully human IgG2 antibody and, in contrast to $\mathrm{Cmab}$, infusion-associated reactions are usually minor in severity and grade 3/4 reactions are rarely experienced. As a result, premedication is not usually recommended when Pmab therapy is administered (4). In our study, Pmab was effective even after the failure of Cmab. In Western countries, there have been a number of previous studies on the safety and clinical efficacy of Pmab following disease progression with $\mathrm{Cmab}$ therapy $(5,6)$. In the present study, $>60 \%$ of patients who experienced disease progression with Cmab therapy achieved varying levels of clinical benefit, including disease control and a reduction in levels of the serum tumor marker CEA, with Pmab therapy. Our results are similar to those reported by Power et al on their clinical experience at the Memorial Sloan-Kettering Cancer Center (6). Notably, we were able to prolong PFS by $\sim 3$ months and the OS by $\sim 8$ months by administering Pmab, even after third-line chemotherapy.

At present, it is not entirely clear why patients who had disease progression on $\mathrm{Cmab}$ were able to derive clinical benefits from Pmab. Recently, Montagut et al (13) revealed that the presence of the acquired EGFR ectodomain mutation (S492R) may provide a molecular explanation for the clinical benefits of Pmab therapy in a subset of patients with mCRC who did not respond to treatment with Cmab. Another possibility is that the two antibodies may inhibit EGFR signaling via separate mechanisms. To explore these possibilities, Freeman et al (14) 
performed epitope mapping of the two antibodies and revealed that Pmab and $\mathrm{Cmab}$ bind to the same surface-exposed amino acids in domain III of EGFRs and this inhibited the binding of all known EGFR ligands. However, formal X-ray crystallographic studies showed that the humanized anti-EGFR antibody matuzumab interacts with an epitope on EGFRs that is distinct from the ligand-binding region on domain III and the Cmab epitope $(15,16)$. Matuzumab indirectly blocked ligand-induced receptor activation by sterically preventing the domain rearrangement and local conformational changes that must occur for high-affinity ligand binding and receptor dimerization. Structural studies of a different humanized anti-EGFR antibody, nimotuzumab, revealed a novel mechanism in which nimotuzumab blocked EGF binding while allowing the EGFR to adopt its active conformation. By interfering with only ligand-dependent EGFR activation, nimotuzumab was able to reduce EGFR signaling to a basal, ligand-independent level. Taken together, these studies suggest that the various anti-EGFR antibodies may inhibit EGFR-mediated signaling through different mechanisms. As a result, it is also conceivable that distinct mechanisms of resistance may develop to the respective anti-EGFR antibodies.

In conclusion, Pmab therapy may represent an alternative treatment strategy for patients, Japanese or otherwise, with refractory $\mathrm{mCRC}$ who have experienced failure with standard therapies, including Cmab-based regimens. Our relatively small clinical experience suggests that $\mathrm{Cmab}$ and $\mathrm{Pmab}$ may exert their antitumor activity via different mechanisms, however, further study is required to investigate this hypothesis.

\section{References}

1. Bokemeyer C, Bondarenko I, Hartmann JT, de Braud F, Schuch G, Zubel A, et al: Efficacy according to biomarker status of cetuximab plus FOLFOX-4 as first-line treatment for metastatic colorectal cancer: the OPUS study. Ann Oncol 22: 1535-1546, 2011.

2. Sobrero AF, Maurel J, Fehrenbacher L, Scheithauer W, Abubakr YA, Lutz MP, et al: EPIC: phase III trial of cetuximab plus irinotecan after fluoropyrimidine and oxaliplatin failure in patients with metastatic colorectal cancer. J Clin Oncol 26 : 2311-2319, 2008.
3. Pfeiffer P, Nielsen D, Yilmaz M, Iversen A, Vejlø C and Jensen BV: Cetuximab and irinotecan as third line therapy in patients with advanced colorectal cancer after failure of irinotecan, oxaliplatin and 5-fluorouracil. Acta Oncol 46: 697-701, 2007.

4. Cohenuram M and Saif MW: Panitumumab the first fully human monoclonal antibody: from the bench to the clinic. Anticancer Drugs 18: 7-15, 2007.

5. Saif MW, Kaley K, Chu E and Copur MS: Safety and efficacy of panitumumab therapy after progression with cetuximab: experience at two institutions. Clinical Colorectal Cancer 9: 315-318, 2010.

6. Power DG, Shah MA, Asmis TR, Garcia JJ and Kemeny NE: Safety and efficacy of panitumumab following cetuximab: retrospective review of the Memorial Sloan-Kettering experience. Invest New Drugs 28: 353-360, 2010.

7. van Persijn van Meerten EL, Gelderblom H and Bloem JL: RECIST revised: implications for the radiologist. A review article on the modified RECIST guideline. Eur Radiol 20: 1456-1467, 2010.

8. Meyerhardt JA and Mayer RJ: Systemic therapy for colorectal cancer. N Engl J Med 352: 476-487, 2005.

9. Van Cutsem E: First-line treatment: approaches with cytotoxic and biologic agents. In: New Treatment Strategies for Metastatic Colorectal Cancer. Chu E (ed). CMP Healthcare Media, Manhasset, NY, pp21-46, 2008.

10. Mendelsohn J and Baselga J: Epidermal growth factor receptor targeting in cancer. Semin Oncol 33: 369-385, 2006.

11. Jean GW and Shah SR: Epidermal growth factor receptor monoclonal antibodies for the treatment of metastatic colorectal cancer. Pharmacotherapy 28: 742-754, 2008.

12. Cmelak AJ, Lordick F, Borner M, Goldberg RM and Saif MW: Management of infusion reactions in clinical trials and beyond: the US and EU perspectives. Oncology (Williston Park) 23 (Suppl 1): 18-25, 2009.

13. Montagut C, Dalmases A, Bellosillo B, Crespo M, Pairet S, Iglesias M, et al: Identification of a mutation in the extracellular domain of the Epidermal Growth Factor Receptor conferring cetuximab resistance in colorectal cancer. Nat Med 18: 221-223, 2012.

14. Freeman D, Sun J, Bass R, et al: Panitumumab and cetuximab epitope mapping and in vitro activity. J Clin Oncol 26 (May 20 Suppl): 14536, 2008.

15. Leahy DJ: A molecular view of anti-ErbB monoclonal antibody therapy. Cancer Cell 13: 291-293, 2008.

16. Schmiedel J, Blaukat A, Li S, Knöchel T and Ferguson KM: Matuzumab binding to EGFR prevents the conformational rearrangement required for dimerization. Cancer Cell 13: 365-373, 2008. 